https://helda.helsinki.fi

\title{
The Relationship Between Body Fat Distribution and \\ Nonalcoholic Fatty Liver in Adults With Type 1 Diabetes
}

\section{FinnDiane Study Grp}

2021-07

FinnDiane Study Grp , Parente , E B , Dahlström , E H, Harjutsalo , V , Inkeri , J , Mutter , S , Forsblom , C , Sandholm , N , Gordin , D \& Groop , P-H 2021, ' The Relationship Between Body Fat Distribution and Nonalcoholic Fatty Liver in Adults With Type 1 Diabetes ' , Diabetes Care, vol. 44 , no. 7 , pp. 1706-1713 . https://doi.org/10.2337/dc20-3175

http://hdl.handle.net/10138/340046

https://doi.org/10.2337/dc20-3175

acceptedVersion

Downloaded from Helda, University of Helsinki institutional repository.

This is an electronic reprint of the original article.

This reprint may differ from the original in pagination and typographic detail.

Please cite the original version. 
Full title

The relationship between body fat distribution and non-alcoholic fatty liver in adults with type 1 diabetes

Short title

Body fat, fatty liver and type 1 diabetes

Authors:

Erika B Parente ${ }^{1,2,3}$, Emma H Dahlström ${ }^{1,2,3}$, Valma Harjutsalo ${ }^{1,2,3,4}$, Jussi Inkeri ${ }^{1,5}$, Stefan Mutter ${ }^{1,2,3}$, Carol Forsblom ${ }^{1,2,3}$, Niina Sandholm ${ }^{1,2,3}$, Daniel Gordin ${ }^{1,2,3,6}$, PerHenrik Groop ${ }^{1,2,3,7}$, on behalf of the FinnDiane Study Group

${ }^{1}$ Folkhälsan Research Center, Helsinki, Finland,

${ }^{2}$ Research Program for Clinical and Molecular Metabolism, Faculty of Medicine, University of Helsinki, Finland,

${ }^{3}$ Abdominal Center, Nephrology, University of Helsinki and Helsinki University Hospital, Helsinki, Finland,

${ }^{4}$ National Institute for Health and Welfare, Helsinki, Finland,

${ }^{5}$ HUS Medical Imaging Center, Radiology, University of Helsinki and Helsinki University Hospital, Helsinki, Finland

${ }^{6}$ Joslin Diabetes Center, Harvard Medical School, Boston, United States of America

${ }^{7}$ Department of Diabetes, Central Clinical School, Monash University, Melbourne, Australia

Number of tables: 3 Number of figures: 1 Word count: 3802

Correspondence author and reprint requests

Per-Henrik Groop. Folkhälsan Research Center, Biomedicum Helsinki, FIN-00014

University of Helsinki, Finland. Tel: $+358-500-430$ 436. E-mail: per-

henrik.groop@helsinki.fi 
Abstract

Objective: Obesity, which is associated with non-alcoholic fatty liver (NAFL), has increased among people with type 1 diabetes. Therefore, we explored the associations between body fat distribution and NAFL in this population.

Research Design and Methods: This study included 121 adults with type 1 diabetes from the Finnish Diabetic Nephropathy Study for whom NAFL was determined by magnetic resonance imaging. Body composition was assessed by dual-energy $\mathrm{x}$-ray absorptiometry. Genetic data concerning PNPLA3 rs738409 and TM6SF2 rs58542926 were available as a directly genotyped polymorphism. Associations between body fat distribution, waist-height ratio (WHtR), body mass index (BMI) and NAFL were explored using logistic regression. A receiver operating characteristic (ROC) curve was used to determine the WHtR and BMI thresholds with the highest sensitivity and specificity to detect NAFL.

Results: Median age was 38.5 (33-43.7) years, duration of diabetes 21.2 (17.9-28.4) years, $52.1 \%$ were women, and the prevalence of NAFL was $11.6 \%$. After adjusting for sex, age, duration of diabetes, and PNPLA3 rs738409, the volume ( $\mathrm{p}=0.03)$ and percentage $(\mathrm{p}=0.02)$ of visceral adipose tissue were associated with NAFL, whereas gynoid, appendicular and total adipose tissues were not. The area under the curve between WHtR and NAFL was larger than BMI and NAFL ( $\mathrm{p}=0.04)$. The WHtR cut-off of 0.5 showed the highest sensitivity (86\%) and specificity (55\%), whereas the BMI of $26.6 \mathrm{~kg} / \mathrm{m}^{2}$ showed $79 \%$ sensitivity and $57 \%$ specificity.

Conclusions: Visceral adipose tissue is associated with NAFL in adults with type 1 diabetes, and WHtR may be considered when screening for NAFL in this population.

Keywords: type 1 diabetes, PNPLA3, TM6SF2, fatty liver, visceral adipose tissue, 
Introduction

Non-alcoholic fatty liver disease (NAFLD) is characterized by excessive accumulation of fat in the liver accompanied by insulin resistance and not related to alcohol consumption greater than $30 \mathrm{~g} / \mathrm{day}$ for men or $20 \mathrm{~g} / \mathrm{day}$ for women (1). It covers a disease spectrum from non-alcoholic fatty liver (NAFL) to non-alcoholic steatohepatitis (NASH), which may progress to fibrosis, cirrhosis, and eventually hepatocellular carcinoma (1-3). NAFLD is typically associated with type 2 diabetes, obesity, and insulin resistance $(1,4-7)$. However, individuals with type 1 diabetes have become more obese during the last decades (8), and NAFLD has also been described in this population (9-12). The prevalence of NAFLD in type 1 diabetes varies from $4.7 \%$ to $50 \%$ depending on age, sex, duration of diabetes, body mass index (BMI), glycaemic control, serum triglycerides as well as on the method used to measure the liver fat content (9-12). Furthermore, NAFLD has been associated with deleterious consequences such as chronic kidney disease (10) and cardiovascular disease in type 1 diabetes (13). Since biomarkers of steatosis have limited clinical utility, as they often do not accurately quantify the percentage of intrahepatic fat content assessed histologically, imaging techniques are the preferred noninvasive diagnostic tools for assessing fat accumulation in the liver. Unfortunately, proton magnetic resonance spectroscopy, the most precise imaging method, is of limited availability owing to its high costs. Therefore, a feasible, accessible, and cost-efficient tool to screen individuals at higher risk of NAFLD is warranted.

Beyond obesity and type 2 diabetes, the missense rs738409 $\mathrm{C}>\mathrm{G}$ single nucleotide polymorphism (SNP) of the PNPLA3 gene, encoding for the patatin-like phospholipase domain-containing protein 3 , is associated with fat accumulation in the liver $(1,14)$. Although the G-allele carriers of rs 738409 do not show increased insulin resistance $(15,16)$, the presence of the G-allele has been associated with severe hepatic outcomes 
such as progressive steatohepatitis, liver fibrosis, and also hepatocarcinoma (17). The variant rs58542926 of the transmembrane 6 superfamily member 2 gene (TM6SF2) is also associated with NAFLD independent of the genetic variant rs738409 in PNPLA3 (14).

Recently, a meta-analysis stressed the importance of central vs. general obesity concerning the risk of all-cause mortality (18). Indeed, visceral adipose tissue has been associated with cardiovascular disease, insulin resistance, and NAFL in people with type 2 diabetes and in the general population $(4,19,20)$. However, the relationship between body fat distribution and NAFL in individuals with type 1 diabetes is unknown. Therefore, in the present study including adults with type 1 diabetes, we aimed to investigate whether the compartments of body adipose tissue are associated with NAFL using logistic regression models adjusted for metabolic and genetic variables. Moreover, since the assessment of body fat distribution requires sophisticated and expensive procedures, such as dual-energy X-ray absorptiometry (DXA), we studied the associations between the waist-height ratio (WHtR), BMI and NAFL, seeking to find an easy and accessible tool for identification of NAFL in this population.

Research Design and Methods

\section{$\underline{\text { Study participants }}$}

All individuals in this study were participants of the Finnish Diabetic Nephropathy (FinnDiane) Study, which is an ongoing, nationwide, prospective, multicenter (93 centers across Finland) study aiming to identify risk factors for type 1 diabetes complications. Type 1 diabetes was defined as age at onset of diabetes under 40 years and permanent insulin treatment initiated within a year from the diabetes diagnosis. During the 
FinnDiane study visit, the participants underwent a thorough clinical examination, collection of blood and urine samples, and completed several questionnaires. From 2011 to 2017,131 individuals attending the Helsinki University Hospital study center were recruited and underwent hepatic magnetic resonance imaging (MRI) to evaluate their liver fat content as part of their FinnDiane study visit. Those with self-reported daily alcohol consumption $\geq 30 \mathrm{~g}$ for men and $\geq 20 \mathrm{~g}$ for women were not included in this study, nor were those with NAFL and missing data on alcohol consumption. In the group without NAFL, individuals with missing data on alcohol consumption $(n=50)$ were included since they did not have NAFL. Finally, 121 individuals were included in the current analysis of NAFL as the outcome. Out of those, 95 individuals had been genotyped for the PNPLA3 SNP rs738409 and the TM6SF2 SNP rs58542926, and 84 individuals had data on body composition available assessed by dual-energy X-ray absorptiometry as part of the Finnish Diabetic Nephropathy Study. The study protocol followed the principles of the Declaration of Helsinki as revised in 2000 and was approved by the Ethical Committee of Helsinki and Uusimaa Hospital District. Written informed consent was obtained from each FinnDiane participant before participation.

\section{$\underline{\text { Liver fat assessment }}$}

Liver fat content was assessed by MRI with a 3.0 T scanner (Achieva; Philips, Best, the Netherlands) at the Helsinki University Hospital Medical Imaging Center. An abdominal radiologist (JI), blinded to all clinical data, evaluated all hepatic MRI exams. We obtained axial images of the liver using gradient-echo T1-weighted, dual-echo, in-phase (IP) and opposed-phase (OP) sequences. Then, three regions of interest (ROI), with $2.00 \mathrm{~cm}^{2}$ each, were drawn at the same location of the liver in both IP and OP images avoiding hepatic vessels on IMPAX picture archiving and communication system (Agfa-Gevaert, Mortsel, Belgium). Finally, the mean value of the three signal intensities was used $(14,21,22)$. The 
hepatic fat fraction was calculated from the equation as follows: dual-echo fat fraction $(\%)=[(\mathrm{IP}-\mathrm{OP}) /(2 \times \mathrm{IP})] \times 100(22)$. NAFL was defined based on a hepatic fat fraction of $\geq 6 \%(11,14)$.

\section{Diabetic kidney disease (DKD) status}

The glomerular filtration rate was estimated (eGFR) using the CKD-EPI formula. Individuals with eGFR $<15 \mathrm{ml} / \mathrm{min} / 1.73 \mathrm{~m}^{2}$, as well as those on dialysis or with kidney transplantation, were not included in this study.

Body composition and anthropometric measures

Body composition was assessed by dual-energy X-ray absorptiometry (DXA, GE Healthcare Lunar version 16, Wisconsin, USA) according to the manufacturer's instructions, and visceral fat was measured by CoreScan (23). The percentages of adipose tissues were related to total body weight. The term appendicular refers to both legs and arms, central body fat refers to android and visceral adipose tissues, whereas peripheral body fat refers to gynoid and appendicular adipose tissues. BMI was calculated by total body weight in kilograms divided by the square of height in meters. Using a stretchresistant tape measure, waist circumference was measured at the horizontal plane midway of the superior iliac crest and the lower margin of the last rib. Hip circumference was measured around the widest part of the great trochanters. The waist-hip ratio was calculated by dividing the waist circumference by the hip circumference. The WHtR was calculated by dividing the waist circumference by the height. Central obesity was defined by a WHtR $\geq 0.5$.

\section{$\underline{\text { Insulin sensitivity and inflammation }}$}

Insulin sensitivity was evaluated using an equation to estimate glucose disposal rate (eGDR) (24) modified for use with $\mathrm{HbA}_{1 c}$ instead of $\mathrm{HbA}_{1}$ (25) while serum high- 
sensitivity C-reactive protein (hsCRP) was used as a surrogate marker of inflammatory status.

\section{Genotyping and genetic variants}

Based on the known association between NAFL and the SNPs rs738409 and rs58542926 $(1,14)$, we retrieved the genotypes from available genome-wide genotyping (GWAS) data on all FinnDiane participants. The QC and genotyping were performed as previously described (26). The SNPs rs738409 and rs58542926 were directly genotyped on the genotyping platform with no missing data for 95 of the 121 study participants. Genotypes for the PNPLA3 rs 738409 were analyzed using an additive model with alleles coded as 0 (CC) and 1 (GC), and 2 (GG) unless otherwise stated. Due to the lower frequency of the TM6SF2 rs58542926, it was not included in the regression models.

\section{$\underline{\text { Statistical analyses }}$}

Descriptive data are presented as mean \pm standard deviation for continuous parametric variables, median (interquartile range) for continuous non-parametric variables, and percentage for categorical variables. For comparison between groups, independent samples t-test, Mann Whitney U-test, and Chi-squared test or Fisher's exact test (when the cells had an expected number below 5) were applied, respectively. We used binary logistic regression analysis to explore the associations between the compartments of body adipose tissue, WHtR, BMI and NAFL as an outcome, adjusted for potential confounders. BMI and WHtR were analyzed as a continuous variable, and WHtR was scaled by a factor of 10 . We limited the number of covariates in each model due to the small number of individuals presenting the outcome. Model 1 was unadjusted. Model 2 was adjusted for unmodifiable risk factors such as age, sex and duration of diabetes. Model 3 was adjusted for unmodifiable risk factors plus $\mathrm{HbA}_{1 \mathrm{c}}$, Model 4 was adjusted for unmodifiable risk 
factors plus triglycerides, and Model 5 was adjusted for unmodifiable risk factors plus the rs738409 (PNPLA3) G allele count. A receiver operating characteristic (ROC) curve was used to graphically show the associations between WHtR, BMI and NAFL as well as to evaluate the sensitivity and specificity of different thresholds of each anthropometric measure. P-values for the differences in area under the curve (AUC) were calculated by a permutation analysis with 10,000 permutations in $\mathrm{R}$ using the pRoc package (27). A two-tailed $P$ value $<0.05$ was considered statistically significant. All data were analyzed using IBM SPSS Statistics for Windows, Version 25.0 (IBM Corp, Armonk, NY, USA) unless otherwise stated. Genotype frequencies were tested for consistency with HardyWeinberg Equilibrium in controls before the analysis, using Fischer's exact test in plink v1.09.

Results

In the total of 121 individuals, the median age was 38.5 (32.3-43.7) years, duration of diabetes was $21.2(17.9-28.4)$ years, $52.1 \%$ were women, $50.4 \%$ presented with central obesity (WHtR $\geq 0.5)$, and the prevalence of NAFL was $11.6 \%(n=14)$.

In total, $78.5 \%(\mathrm{n}=95)$ of the 121 included individuals had genetic data available for the PNPLA3 rs738409 and the TM6SF2 rs58542926 SNPs. The genotypes did not deviate from the Hardy-Weinberg Equilibrium (rs738409; $\mathrm{p}=0.11$ and rs58542926; $\mathrm{p}=0.99$ ) and, the minor allele frequency was $18.4 \%$ (G-allele) for rs738409 and $6.3 \%$ (T-allele) for rs58542926. In total, 28 individuals were either homozygotes (GG, $n=7)$ or heterozygotes (CG, $\mathrm{n}=21$ ) for the minor G-allele of PNPLA3 rs738409 and the minor allele frequency was $32.1 \%$ among cases and $16.1 \%$ among controls. Using an additive model, the odds ratio (OR) for NAFL was $2.48(\mathrm{p}=0.06)$. Concerning TM6SF2 rs58542926, none of the 
participants was homozygous (TT) for the minor T-allele, 12 individuals were heterozygous (TC) and none of them was in the group with NAFL. As the number of Tallele carrying participants was limited, the SNP was excluded from further analyses. The distribution of individuals with the SNPs rs738409 and rs58542926 according to the presence of NAFL is depicted in Table 1.

Individuals with NAFL had a longer duration of diabetes, higher $\mathrm{HbA}_{1 \mathrm{c}}$ and triglycerides than those without NAFL. Moreover, they had lower insulin sensitivity based on the lower eGDR $(3.1 \mathrm{mg} / \mathrm{kg} / \mathrm{min}$ vs. $7.6 \mathrm{mg} / \mathrm{kg} / \mathrm{min}, \mathrm{p}<0.001)$ and higher daily insulin requirement per kilogram of body weight ( $0.76 \mathrm{IU} / \mathrm{kg}$ vs. $0.52 \mathrm{IU} / \mathrm{kg}, \mathrm{p}=0.026)$ (Table 1$)$. More people in the NAFL group were centrally obese ( $85.7 \%$ in vs. $45.8 \%, \mathrm{p}=0.005)$ compared to the group without NAFL. Interestingly, the percentage of total, appendicular or gynoid adipose tissues did not differ between the individuals with or without NAFL (Table 2). Nevertheless, those with NAFL presented with higher percentages of android (3.47\% vs. $2.40 \%, \mathrm{p}=0.02)$ and visceral $(1.83 \%$ vs. $0.55 \%, \mathrm{p}=0.01)$ adipose tissues compared to those without NAFL (Table 2).

By using logistic regression models to explore whether the compartments of body adipose tissues are associated with NAFL, we found that the volume and percentage of visceral adipose tissue were positively associated with NAFL not only in the unadjusted model but also after adjusting for covariates (Table 3). In the unadjusted model, for each one percent increase in visceral adipose tissue, the odds of NAFL increased 4.6-fold $(\mathrm{p}=0.001)$ (Table 3). However, the percentages of appendicular, gynoid, and total adipose tissues were not associated with NAFL (Table 3).

We used logistic regression models to evaluate the association between WHtR, BMI and NAFL. Similarly to the visceral adipose tissue, the increase in WHtR was positively associated with NAFL in the unadjusted model (OR 7.59, $\mathrm{p}<0.001)$, and this association 
remained after adjusting for sex, age and duration of diabetes (OR 7.50, $\left.\mathrm{p}=1.40 \times 10^{-4}\right)$, and additional adjustments for $\mathrm{HbA}_{1 \mathrm{c}}\left(\mathrm{OR} 6.68, \mathrm{p}=4.47 \times 10^{-4}\right)$, or triglycerides $(\mathrm{OR} 5.12$, $\mathrm{p}=0.003$ ), or the PNPLA3 SNP rs738409 (OR 6.64, $\mathrm{p}<0.001)$. BMI was associated with NAFL (OR 1.21, $\mathrm{p}=0.002$ ) in the unadjusted model, after adjusting for sex, age and duration of diabetes (OR 1.22, $\mathrm{p}=0.004)$, and additional adjustments for $\mathrm{HbA}_{1 \mathrm{c}}(\mathrm{OR} 1.19$, $\mathrm{p}=0.015$ ), or triglycerides (OR 1.16, $\mathrm{p}=0.045)$, or the PNPLA3 SNP rs738409 (OR 1.22, $\mathrm{p}=0.004$ ). According to the ROC curve, we found that the commonly used WHtR threshold of 0.5 was the best cut-off to detect NAFL in this population with an $86 \%$ sensitivity and $55 \%$ specificity. The BMI of $26.6 \mathrm{~kg} / \mathrm{m}^{2}$ was the best cut-off with a $79 \%$ sensitivity and $57 \%$ specificity. The well-known BMI cut-off of $25 \mathrm{~kg} / \mathrm{m}^{2}$ showed a sensitivity of $86 \%$ and specificity of $43 \%$, whereas the BMI of $30 \mathrm{~kg} / \mathrm{m}^{2}$ showed a $43 \%$ sensitivity and $81 \%$ specificity. The AUC of the association between WHtR and NAFL [0.823, CI 95\% (0.692-0.955), $\mathrm{p}<0.001)]$ was larger $(\mathrm{p}=0.04)$ than the AUC of the association between BMI and NAFL [0.720, CI 95\% (0.572-0.955), $\mathrm{p}<0.007)$ ] (Figure 1).

\section{Conclusions}

The main finding of this study is that the visceral adipose tissue, but not the total or the peripheral body fat (appendicular and gynoid adipose tissues), is associated with NAFL in adults with type 1 diabetes. Furthermore, we showed that WHtR, a simple and lowcost surrogate marker of visceral adipose tissue, is strongly associated with NAFL and could be used as a screening tool for NAFL in this population.

Although NAFL has often been linked to obesity in the general population, and in individuals with type 2 diabetes $(1,28)$, its presence in individuals with type 1 diabetes is not negligible. A previous study in individuals with type 1 diabetes has shown a 
prevalence of up to $50 \%$ of NAFL when assessed by ultrasound (10). This number is considerably higher than the prevalence found in our cohort $(11.6 \%)$ or in two other studies in which MRI was used to assess the liver fat content $(9,11)$. The differences in prevalence are most likely explained by the different methodologies to measure the liver fat content (21). The prevalence of NAFL found in our study was lower than the prevalence of NAFL in type 2 diabetes or the general population $(1,11)$, possibly because individuals with type 1 diabetes do not have insulin delivery from the pancreas into the portal system acting directly on the liver insulin receptors and thereby stimulating lipogenesis (7). In line with this hypothesis is the $8.8 \%$ prevalence of NAFL in American individuals with type 1 diabetes compared to $75.6 \%$ of Americans with type 2 diabetes as shown in the American study by Cusi et al (11), whereas the prevalence of NAFL in the American general population varies from 19 to $46 \%(1,11)$.

In the current study, the individuals with NAFL showed more signs of chronic inflammation (higher hsCRP) and insulin resistance (lower eGDR and higher daily insulin requirement per kilogram of body weight), suggesting that these individuals may have NAFLD, which encompass insulin resistance and inflammation beyond steatosis. The harmful consequences of NAFLD go beyond the liver and are associated with cardiovascular disease in the general population, in type 2 diabetes $(12,28)$, and also in people with type 1 diabetes (13). However, the answer to the question whether the lowgrade chronic inflammation together with the lower insulin sensitivity found in our study contributes to the progression of NAFL and/or cardiovascular outcomes requires future longitudinal studies.

The inflammatory status and low insulin sensitivity found in our population are possibly a consequence of the higher volume and percentage of visceral adipose tissue in those 
with NAFL compared to those without, given that an increase in visceral adipose tissue is closely associated with chronic inflammation and insulin resistance $(19,29)$.

In the present study, we observed that the associations between the visceral adipose tissue and the NAFL were still significant after adjusting for age, sex, duration of diabetes, $\mathrm{HbA}_{1 \mathrm{c}}$, or triglycerides. In addition, we explored whether genetics could be a confounder, since the SNP rs738409 in PNPLA3 has been linked to NAFL $(1,14)$. The visceral adipose tissue was associated with NAFL even after adjusting for sex, age, duration of diabetes, and the SNP. Furthermore, a similar association by using unadjusted and adjusted models was seen between NAFL and WHtR, which is a surrogate marker of visceral adipose tissue (30). Interestingly, the liver fat accumulation associated with the SNP is not linked to insulin resistance $(15,16)$, but the individuals with NAFL in our cohort presented with lower insulin sensitivity than those without NAFL, suggesting that NAFL may be a consequence of excess of visceral adipose tissue rather than genetics.

In contrast to a previous publication (31), our results suggest that individuals with type 1 diabetes are not protected from NAFL just because they do not have portal insulin acting directly on the liver insulin receptors and activating the glycogen synthesis and de novo lipogenesis (7). However, peripheral insulin indirectly regulates the hepatic glucose and lipid metabolism by inhibiting adipose lipolysis and promoting muscle glucose uptake (7). Therefore, individuals with type 1 diabetes may accumulate fat in the liver as long as they are centrally obese and insulin resistant. On the other hand, the increased fat deposition in the liver can also lead to insulin resistance in the liver, which in turn would increase the hepatic glucose output and contribute to hyperglycaemia and dyslipidemia $(6,7)$, and thereby maintaining the cycle of insulin resistance and metabolic disturbances.

We also found that the total and peripheral body fat were not associated with NAFL. Notably, these findings reflect the different metabolic functions of the adipocytes in 
different adipose tissue compartments, such as visceral and subcutaneous adipose tissues $(32,33)$. Another example of the differences between adipose tissue compartments concerns the android adipose tissue, which is composed of visceral and subcutaneous adipose tissues located in the central region of the body. In the current study, the android adipose tissue was associated with NAFL in the unadjusted model and after adjusting for age, sex, and duration of diabetes, but not after adjusting for $\mathrm{HbA}_{1 \mathrm{c}}$, or triglycerides, or the SNP rs738409 in PNPLA3. On the other hand, the visceral adipose tissue was still associated with NAFL after all adjustments, suggesting the visceral adipose tissue is crucial for the accumulation of fat in the liver. The impact of the android adipose tissue on NAFL was probably attenuated by the presence of subcutaneous fat.

Although peripheral body fat has been proposed as a protective adipose tissue concerning metabolic diseases $(6,33)$, in our study, it was not protective of NAFL. The individuals with NAFL presented similar percentages of appendicular and gynoid adipose tissues but higher percentages of visceral and android adipose tissues than those without NAFL, which means that the central fat distribution is possibly behind the results. The higher prevalence of central obesity in individuals with NAFL could also be related to sex. However, there was no difference in sex distribution between the two groups. Additionally, we included sex as a covariate in all models of the logistic regression to mitigate this issue.

The reason why some individuals with central obesity develop NAFL, and some do not, is still unclear and cannot be answered by our cross-sectional study. However, interactions between genetic variants and body fat distribution $(34,35)$ and liver fat accumulation (14) is a possible hypothesis to be investigated in future studies.

Finally, considering that the assessment of visceral adipose tissue requires costly imaging procedures such as DXA, we showed in this study that a simple measure such as WHtR 
is strongly associated with NAFL and may assist in screening individuals with type 1 diabetes at higher risk of NAFL for further referral to imaging evaluation. This is in line with results in the general Finnish population in which the WHtR showed a hazard ratio of 1.44 (95\% CI 1.12-1.87) for the incidence of NAFLD (36). The AUC of the ROC curve for the association between WHtR and NAFL in our cohort was 0.823 (CI 95\%, 0.6920.955), which is similar to a previous publication including individuals without diabetes [AUC 0.878 (95\% CI 0.82 to 0.94)]. These findings suggest that WHtR is a reliable tool for screening NAFL (37). Furthermore, according to our results, both measures WHtR and BMI were associated with NAFL. However, WHtR showed a stronger association with NAFL than BMI, which is in line with the association between central body fat and NAFL, but not between peripheral body fat and NAFL. If BMI would be used as a screening tool, the suggested cut-off would be $26.6 \mathrm{~kg} / \mathrm{m}^{2}$ which showed a similar specificity compared to the WHtR cut-off of 0.5 , although with lower sensitivity.

A limitation of the present study is the absence of serum hepatic enzymes and platelets that would have enabled the calculation of a clinical score of fibrosis, as well as the estimation of hepatocyte injury. However, the lack of this information did not impact our results since we aimed to study the association between body fat distribution and NAFL, not NASH. The lack of dietary and physical activity information is a shortcoming as the diet components could have had an impact on NAFL and the body composition. Finally, the cross-sectional study limits any inferences to causal relationships. Nevertheless, the study has several strengths. The individuals were thoroughly examined, the liver fat content was measured by MRI and the body composition by DXA, which are gold standard methods. Another strength is that the SNPs rs738409 in PNPLA3 and TM6SF2 rs58542926 were available as directly genotyped SNPs in the GWAS with no missing data for 95 of the 121 study participants. Overall, our results motivate further studies to 
explore possible mechanisms and genetic variants, involved in the relationship between body fat distribution and ectopic fat deposits in the liver of people with type 1 diabetes.

From a clinical point of view, beyond showing that the visceral adipose tissue is associated with NAFL in adults with type 1 diabetes, we also show that WHtR may be useful as an easy and inexpensive tool to screen individuals at higher risk of NAFL. Then, future studies will show the cost-effectiveness of this tool. Finally, considering the recent publication regarding the effect of liraglutide on the reduction of adipose tissue and visceral fat in type 1 diabetes (38), our results raise a question to be answered by future clinical trials whether individuals with type 1 diabetes, central obesity, and NAFL should receive pharmacological treatment for obesity and insulin resistance, beyond lifestyle recommendations and insulin therapy.

In conclusion, our study shows that individuals with type 1 diabetes are not protected from NAFL and that visceral adipose tissue is associated with NAFL after adjusting for confounders, including the missense SNP rs738409 in the PNPLA3 gene. Furthermore, the WHtR may be considered as a screening tool for NAFL in this population.

Acknowledgements

$\underline{\text { Author contributions }}$

EBP was responsible for the concept and study design, statistical analyses, and preparation of the manuscript. EHD and NS were responsible for the acquisition and analysis of genetic data. JI analyzed the liver MRI images. VH, CF and DG contributed to the acquisition of the clinical data. All authors interpreted the results and contributed to the critical revision of the manuscript. PHG is the guarantor of this work and takes full 
responsibility for the integrity of the data and the accuracy of the data analysis. All authors reviewed the manuscript and approved the final version.

The authors would like to acknowledge A. Sandelin, J. Tuomikangas and M. Korolainen for their technical assistance. We are indebted to Drs. Turgut Tatlisumak and Jukka Putaala for facilitating liver MRi imaging.

Funding

This research was funded by grants from Folkhälsan Research Foundation, Academy of Finland (299200, 316664 and UAK10121MRI), Wilhelm and Else Stockmann Foundation, Sigrid Juselius Foundation, Liv och Hälsa Society, Finska Läkaresällskapet (Medical Society of Finland), Diabetes Research Foundation, Novo Nordisk Foundation (NNF OC0013659), Päivikki and Sakari Sohlberg Foundation, EVO governmental grants, Dorothea Olivia, Karl Walther och Jarl Walther Perklén Foundation, and University of Helsinki (Clinical Researcher stint for DG).

\section{$\underline{\text { Conflict of interest statement }}$}

EBP reports receiving lecture honorariums from Eli Lilly, Abbott, Astra Zeneca, Sanofi, Boehringer Ingelheim and is an advisory board member of Sanofi. D.G. has received lecture or advisory honoraria from AstraZeneca, Boehringer Ingelheim, Fresenius, GE Healthcare, and Novo Nordisk, and support to attend medical meetings from CVRx and Sanofi Aventis. P-H.G. reports receiving lecture honorariums from Astellas, Astra Zeneca, Boehringer Ingelheim, Eli Lilly, Elo Water, Medscape, MSD, Mundipharma, Novo Nordisk, PeerVoice, Sanofi, Sciarc, and being an advisory board member of AbbVie, Astellas, Astra Zeneca, Bayer, Boehringer Ingelheim, Eli Lilly, Medscape, MSD, Mundipharma, Novo Nordisk, and Sanofi. SM, VH, AJA, JI and CF report no conflict of interest. 
References:

1. European Association for the Study of the Liver (EASL), European Association for the Study of Diabetes (EASD), European Association for the Study of Obesity (EASO). EASL-EASD-EASO Clinical Practice Guidelines for the management of non-alcoholic fatty liver disease. J Hepatol. 2016;64(6):1388-402.

2. Yki-Järvinen H. Non-alcoholic fatty liver disease as a cause and a consequence of metabolic syndrome. Lancet Diabetes Endocrinol. 2014 Nov;2(11):901-10.

3. Bellentani S. The epidemiology of non-alcoholic fatty liver disease. Liver Int. 2017;37(S1):81-4.

4. Samuel VT, Shulman GI. Nonalcoholic Fatty Liver Disease, Insulin Resistance, and Ceramides. N Engl J Med. 2019 Nov 7;381(19):1866-9.

5. Perry RJ, Samuel VT, Petersen KF, Shulman GI. The role of hepatic lipids in hepatic insulin resistance and type 2 diabetes. Nature. 2014 Jun 5;510(7503):8491 .

6. Godoy-Matos AF, Silva Júnior WS, Valerio CM. NAFLD as a continuum: from obesity to metabolic syndrome and diabetes. Diabetol Metab Syndr. 2020 Jul $14 ; 12: 60$.

7. Samuel VT, Shulman GI. Nonalcoholic Fatty Liver Disease as a Nexus of Metabolic and Hepatic Diseases. Cell Metab. 2018 Jan 9;27(1):22-41.

8. Dahlström EH, Sandholm N, Forsblom CM, Thorn LM, Jansson FJ, Harjutsalo V, et al. Body Mass Index and Mortality in Individuals With Type 1 Diabetes. J Clin Endocrinol Metab. 2019 Nov 1;104(11):5195-204.

9. Petit J-M, Pedro L, Guiu B, Duvillard L, Bouillet B, Jooste V, et al. Type 1 diabetes is not associated with an increased prevalence of hepatic steatosis. Diabet Med. 2015 Dec;32(12):1648-51.

10. Targher G, Mantovani A, Pichiri I, Mingolla L, Cavalieri V, Mantovani W, et al. Nonalcoholic Fatty Liver Disease Is Independently Associated With an Increased Incidence of Chronic Kidney Disease in Patients With Type 1 Diabetes. Diabetes Care. 2014 Jun;37(6):1729-36.

11. Cusi K, Sanyal AJ, Zhang S, Hartman ML, Bue-Valleskey JM, Hoogwerf BJ, et al. Non-alcoholic fatty liver disease (NAFLD) prevalence and its metabolic associations in patients with type 1 diabetes and type 2 diabetes. Diabetes Obes Metab. 2017 Nov;19(11):1630-4.

12. Targher G, Bertolini L, Padovani R, Rodella S, Tessari R, Zenari L, et al. Prevalence of nonalcoholic fatty liver disease and its association with cardiovascular disease among type 2 diabetic patients. Diabetes Care. 2007 May;30(5):1212-8. 
13. Targher G, Bertolini L, Padovani R, Rodella S, Zoppini G, Pichiri I, et al. Prevalence of non-alcoholic fatty liver disease and its association with cardiovascular disease in patients with type 1 diabetes. J Hepatol. 2010 Oct;53(4):713-8.

14. Petäjä EM, Yki-Järvinen H. Definitions of Normal Liver Fat and the Association of Insulin Sensitivity with Acquired and Genetic NAFLD-A Systematic Review. Int J Mol Sci. 2016 Apr 27;17(5):633.

15. Franko A, Merkel D, Kovarova M, Hoene M, Jaghutriz BA, Heni M, et al. Dissociation of Fatty Liver and Insulin Resistance in I148M PNPLA3 Carriers: Differences in Diacylglycerol (DAG) FA18:1 Lipid Species as a Possible Explanation. Nutrients. 2018 Sep 17;10(9):1314.

16. Kantartzis K, Peter A, Machicao F, Machann J, Wagner S, Königsrainer I, et al. Dissociation Between Fatty Liver and Insulin Resistance in Humans Carrying a Variant of the Patatin-Like Phospholipase 3 Gene. Diabetes. 2009 Nov;58(11):2616-23.

17. Liu Y-L, Patman GL, Leathart JBS, Piguet A-C, Burt AD, Dufour J-F, et al. Carriage of the PNPLA3 rs738409 $\mathrm{C}>\mathrm{G}$ polymorphism confers an increased risk of non-alcoholic fatty liver disease associated hepatocellular carcinoma. J Hepatol. 2014 Jul;61(1):75-81.

18. Jayedi A, Soltani S, Zargar MS, Khan TA, Shab-Bidar S. Central fatness and risk of all cause mortality: systematic review and dose-response meta-analysis of 72 prospective cohort studies. BMJ. 2020 Sep 23;370:m3324.

19. Després J-P. Health consequences of visceral obesity. Ann Med. 2001 Nov;33(8):534-41.

20. Kang SH, Cho KH, Park JW, Yoon KW, Do JY. Association of Visceral Fat Area with Chronic Kidney Disease and Metabolic Syndrome Risk in the General Population: Analysis Using Multi-Frequency Bioimpedance. Kidney Blood Press Res. 2015;40(3):223-30.

21. Mashhood A, Railkar R, Yokoo T, Levin Y, Clark L, Fox-Bosetti S, et al. Reproducibility of hepatic fat fraction measurement by magnetic resonance imaging. J Magn Reson Imaging. 2013 Jun;37(6):1359-70.

22. Shin HJ, Kim HG, Kim M-J, Koh H, Kim HY, Roh YH, et al. Normal Range of Hepatic Fat Fraction on Dual- and Triple-Echo Fat Quantification MR in Children. PLoS One. 2015 Feb 6;10(2):e0117480.

23. Meredith-Jones K, Haszard J, Stanger N, Taylor R. Precision of DXA-Derived Visceral Fat Measurements in a Large Sample of Adults of Varying Body Size. Obesity (Silver Spring). 2018 Mar;26(3):505-12.

24. Williams KV, Erbey JR, Becker D, Arslanian S, Orchard TJ. Can clinical factors estimate insulin resistance in type 1 diabetes? Diabetes. 2000 Apr;49(4):626-32. 
25. Thorn LM, Forsblom C, Fagerudd J, Thomas MC, Pettersson-Fernholm K, Saraheimo M, et al. Metabolic Syndrome in Type 1 Diabetes: Association with diabetic nephropathy and glycemic control (the FinnDiane study). Diabetes Care. 2005 Aug 1;28(8):2019-24.

26. Syreeni A, Sandholm N, Cao J, Toppila I, Maahs DM, Rewers MJ, et al. Genetic Determinants of Glycated Hemoglobin in Type 1 Diabetes. Diabetes. 2019 Apr;68(4):858-67.

27. Robin X, Turck N, Hainard A, Tiberti N, Lisacek F, Sanchez J-C, et al. pROC: an open-source package for $\mathrm{R}$ and $\mathrm{S}+$ to analyze and compare ROC curves. BMC Bioinformatics. 2011 Mar 17;12(1):77.

28. Kasper P, Martin A, Lang S, Kütting F, Goeser T, Demir M, et al. NAFLD and cardiovascular diseases: a clinical review. Clin Res Cardiol. 2020 Jul 21;

29. Bertin E, Nguyen P, Guenounou M, Durlach V, Potron G, Leutenegger M. Plasma levels of tumor necrosis factor-alpha (TNF-alpha) are essentially dependent on visceral fat amount in type 2 diabetic patients. Diabetes Metab. 2000 May;26(3):178-82.

30. Parente EB, Mutter S, Harjutsalo V, Ahola AJ, Forsblom C, Groop P-H. Waistheight ratio and waist are the best estimators of visceral fat in type 1 diabetes. Sci Rep. 2020 Oct 29;10(1):18575.

31. Llauradó G, Sevastianova K, Sädevirta S, Hakkarainen A, Lundbom N, OrhoMelander M, et al. Liver fat content and hepatic insulin sensitivity in overweight patients with type 1 diabetes. J Clin Endocrinol Metab. 2015 Feb;100(2):607-16.

32. Halberg N, Wernstedt I, Scherer PE. The Adipocyte as an Endocrine Cell. Endocrinol Metab Clin North Am. 2008 Sep;37(3):753-xi.

33. Wajchenberg BL. Subcutaneous and visceral adipose tissue: their relation to the metabolic syndrome. Endocr Rev. 2000 Dec;21(6):697-738.

34. Li X, Qi L. Gene-Environment Interactions on Body Fat Distribution. Int J Mol Sci. 2019 Jul 27;20(15):3690.

35. Fehlert E, Wagner R, Ketterer C, Böhm A, Machann J, Fritsche L, et al. Genetic determination of body fat distribution and the attributive influence on metabolism. Obesity (Silver Spring). 2017 Jul;25(7):1277-83.

36. Åberg F, Jula A. The sagittal abdominal diameter: Role in predicting severe liver disease in the general population. Obes Res Clin Pract. 2018 Aug;12(4):394-6.

37. Zheng R-D, Chen Z-R, Chen J-N, Lu Y-H, Chen J. Role of Body Mass Index, Waist-to-Height and Waist-to-Hip Ratio in Prediction of Nonalcoholic Fatty Liver Disease. Gastroenterol Res Pract. 2012;2012:362147.

38. Ghanim H, Batra M, Green K, Abuaysheh S, Hejna J, Makdissi A, et al. Liraglutide treatment in overweight and obese patients with type 1 diabetes: A 26- 
week randomized controlled trial; mechanisms of weight loss. Diabetes Obes Metab. 2020 Oct;22(10):1742-52. 
Table 1. Clinical characteristics and genetic data of participants according to NAFL

\begin{tabular}{|c|c|c|c|}
\hline \multirow[b]{2}{*}{$\mathrm{n}(\%)$} & NAFL (-) & NAFL $(+)$ & $\mathrm{p}$-value \\
\hline & $107(88.4)$ & $14(11.6)$ & \\
\hline Women $(\%)$ & 54.2 & 35.7 & 0.19 \\
\hline Age (year) & $37.8(32.6-43.3)$ & $42.8(31.4-46.7)$ & 0.23 \\
\hline Age at onset DM (year) & $14.2(8.6-22.8)$ & $8.1(4.8-25.0)$ & 0.11 \\
\hline Duration of DM (year) & $20.6(17.7-27.3)$ & $27.8(19.6-32.7)$ & 0.049 \\
\hline Systolic blood pressure (mmHg) & $129 \pm 14$ & $135 \pm 17$ & 0.16 \\
\hline Diastolic blood pressure (mmHg) & $77 \pm 9$ & $82 \pm 11$ & 0.06 \\
\hline Total-C (mmol/L) & $4.34(3.96-4.91)$ & $4.65(3.76-5.80)$ & 0.35 \\
\hline HDL-C (mmol/L) & $1.47 \pm 0.36$ & $1.34 \pm 0.44$ & 0.20 \\
\hline LDL-C (mmol/L) & $2.60(2.27-3.18)$ & $2.84(2.17-4.04)$ & 0.47 \\
\hline $\mathrm{TG}(\mathrm{mmol} / \mathrm{L})$ & $0.86(0.70-1.20)$ & $2.05(1.13-2.60)$ & $<0.001$ \\
\hline hs-CRP (mg/L) & $1.22(0.49-3.03)$ & $3.84(1.34-7.81)$ & 0.002 \\
\hline $\mathrm{HbA1c}(\mathrm{mmol} / \mathrm{L})$ & $63.9 \pm 12.7$ & $74.9 \pm 9.8$ & 0.002 \\
\hline HbA1c (\%) & $8.0 \pm 1.2$ & $9.0 \pm 0.9$ & 0.002 \\
\hline Daily insulin (IU/kg body weight) & $0.52(0.39-0.66)$ & $0.75(0.50-0.91)$ & 0.026 \\
\hline eGDR (mg/kg/min) & $7.6(4.8-9.2)$ & $3.1(2.1-4.5)$ & $<0.001$ \\
\hline Liver fat fraction $(\%)$ & $0.8(0.0-3.9)$ & $10.5(6.7-11.8)$ & $<0.001$ \\
\hline Alcohol consumption (g/day) & $8.6(3.4-16.3)$ & $6.9(0.0-12.9)$ & 0.24 \\
\hline eGFR (ml/min/1.73 m²) & $109(98-116)$ & $112(105-122)$ & 0.22 \\
\hline \multicolumn{4}{|l|}{ Anthropometric measures } \\
\hline Weight (kg) & $80.4 \pm 14.2$ & $92.5 \pm 24.3$ & 0.09 \\
\hline Height $(\mathrm{cm})$ & $174.1 \pm 9.7$ & $172.3 \pm 9.8$ & 0.52 \\
\hline $\operatorname{BMI}\left(\mathrm{kg} / \mathrm{m}^{2}\right)$ & $26.5 \pm 4.0$ & $30.8 \pm 6.3$ & 0.024 \\
\hline Waist (cm) & $87.7 \pm 10.8$ & $105.1 \pm 18.9$ & 0.005 \\
\hline WHR & $0.86 \pm 0.07$ & $0.98 \pm 0.08$ & $<0.001$ \\
\hline WHtR & $0.49(0.47-0.55)$ & $0.60(0.53-0.68)$ & $<0.001$ \\
\hline WHtR $\geq 0.5(\%)$ & 45.8 & 85.7 & 0.005 \\
\hline Genetics & $\mathrm{n}=81$ & $\mathrm{n}=14$ & \\
\hline PNPLA3 & & & 0.09 \\
\hline $\mathrm{CC}(\%)$ & 72.8 & 57.2 & \\
\hline $\mathrm{CG}(\%)$ & 22.2 & 21.4 & \\
\hline GG $(\%)$ & 5.0 & 21.4 & \\
\hline TM6SF2 & & & 0.20 \\
\hline $\mathrm{CC}(\%)$ & 85.2 & 100 & \\
\hline $\mathrm{TC}(\%)$ & 14.8 & 00 & \\
\hline $\mathrm{TT}(\%)$ & 00 & 00 & \\
\hline $\begin{array}{l}\text { Data are shown as per } \\
\text { distributed continuous } \\
\text { distribution. Between-g } \\
\text { the cells had an expe } \\
\text { respectively. In the NA } \\
\text { data. Total-C: total c } \\
\text { lipoprotein cholesterol; } \\
\text { haemoglobin A1c; eG } \\
\text { estimated glomerular f } \\
\text { ratio, PNPLA3: patatin } \\
\text { member } 2 \text {. }\end{array}$ & $\begin{array}{l}\text { categorical varial } \\
\text { nd mean } \pm \text { standa } \\
\text { arisons were done } \\
r \text { below } 5 \text {, Mann } \\
\text {, there were } 50 \text { out } \\
\text { HDL-C: high-den } \\
\text { erides; hs-CRP: h } \\
\text { ted glucose dispo } \\
\text {, BMI: body mass } \\
\text { lolipase domain-co }\end{array}$ & $\begin{array}{l}\text { (interquartile range } \\
\text { for continuous var } \\
\text { square test or Fishe } \\
\text { est, and independ } \\
\text { uals with missing a } \\
\text { in cholesterol; L } \\
\text { C-reactive proteil } \\
\text { FL: non-alcoholic } \\
\text { : waist-hip ratio, } \\
\text { M6SF2: transmemb }\end{array}$ & $\begin{array}{l}\text { n-normally } \\
\text { ith norma } \\
\text { t test when } \\
\text { les' t-test } \\
\text { nsumption } \\
\text { ow-density } \\
\text { c: glycated } \\
\text { er, eGFR } \\
\text { aist-heigh } \\
\text { uperfamily }\end{array}$ \\
\hline
\end{tabular}


Table 2. Body composition of participants according to NAFL

\begin{tabular}{lccc}
\hline & NAFL (-) & NAFL (+) & p-value \\
\cline { 2 - 4 } Body composition & $\mathrm{n}=74$ & $\mathrm{n}=10$ & 0.15 \\
\hline Total adipose tissue $(\mathrm{kg})$ & $24.50 \pm 8.75$ & $28.88 \pm 10.50$ & 0.35 \\
Total adipose tissue (\%) & $31.31 \pm 8.52$ & $33.93 \pm 6.78$ & 0.85 \\
Appendicular adipose tissue (kg) & $11.05 \pm 4.00$ & $10.80 \pm 3.06$ & 0.14 \\
Appendicular adipose tissue (\%) & $14.32 \pm 4.54$ & $3.89(3.16-4.94)$ & 0.76 \\
Gynoid adipose tissue (kg) & $4.35(3.18-5.09)$ & $5.13(4.48-5.31)$ & 0.30 \\
Gynoid adipose tissue (\%) & $5.75(3.91-6.94)$ & $3.04(1.45-4.06)$ & 0.033 \\
Android adipose tissue (kg) & $1.90(1.38-2.48)$ & $3.47(2.35-4.53)$ & 0.023 \\
Android adipose tissue $(\%)$ & $2.40(1.99-3.08)$ & $1.60(0.26-3.02)$ & 0.013 \\
Visceral adipose tissue (kg) & $0.43(0.18-0.99)$ & $1.83(0.41-3.03)$ & 0.012 \\
Visceral adipose tissue $(\%)$ & $0.55(0.25-1.13)$ & $1693(279-3196)$ & 0.013 \\
Visceral adipose tissue $\left(\mathrm{cm}^{3}\right)$ & $452(186-1055)$ & & \\
\hline
\end{tabular}

Data are shown as median (interquartile range) for non-normally distributed variables and mean \pm standard deviation for variables with normal distribution. Between-group comparisons were done with Mann Whitney U-test and independent samples' t-test, respectively. Appendicular means both arms and legs. The percentages of body composition are related to total body weight. 
Table 3. Associations between body fat distribution and NAFL

\begin{tabular}{|c|c|c|c|c|c|c|c|c|c|c|}
\hline & Model 1 & & Model 2 & & Model 3 & & Model 4 & & Model 5 & \\
\hline & OR $(95 \% \mathrm{CI})$ & $\mathrm{p}$-value & OR $(95 \% \mathrm{CI})$ & p-value & OR $(95 \% \mathrm{CI})$ & $\mathrm{p}$-value & OR $(95 \% \mathrm{CI})$ & $\mathrm{p}$-value & OR $(95 \% \mathrm{CI})$ & $\mathrm{p}$-value \\
\hline TAT (\%) & $1.04(0.96-1.13)$ & 0.35 & $1.07(0.95-1.21)$ & 0.24 & $1.09(0.96-1.23)$ & 0.18 & $1.01(0.89-1.15)$ & 0.86 & $1.07(0.95-1.22)$ & 0.27 \\
\hline AppAT & $0.93(0.79-1.09)$ & 0.36 & $0.93(0.70-1.22)$ & 0.58 & $0.98(0.71-1.34)$ & 0.89 & $0.94(0.70-1.25)$ & 0.66 & $0.93(0.69-1.24)$ & 0.61 \\
\hline GAT (\%) & $0.83(0.56-1.24)$ & 0.36 & $0.74(0.36-1.52)$ & 0.41 & $0.86(0.38-1.97)$ & 0.73 & $0.70(0.32-1.54)$ & 0.38 & $0.78(0.36-1.66)$ & 0.51 \\
\hline $\operatorname{AAT}(\%)$ & $2.41(1.22-4.74)$ & 0.01 & $2.34(1.04-5.28)$ & 0.04 & $2.36(1.00-5.53)$ & 0.04 & $1.60(0.65-3.95)$ & 0.31 & $2.05(0.93-4.54)$ & 0.07 \\
\hline $\operatorname{VAT}(\%)$ & $4.63(1.83-11.67)$ & 0.001 & $4.77(1.46-15.57)$ & 0.01 & $4.17(1.12-15.51)$ & 0.03 & $3.88(0.98-15.37)$ & 0.05 & $4.09(1.22-13.74)$ & 0.02 \\
\hline
\end{tabular}

Associations were calculated using a binary logistic regression model with NAFL as the outcome. Model 1 was unadjusted; Model 2 was adjusted for unmodifiable risk factors such as age, sex and duration of diabetes; Model 3 was adjusted for unmodifiable risk factors plus HbA $\mathrm{A}_{1 \mathrm{c}}$; Model 4 was adjusted for unmodifiable risk factors plus triglycerides, and Model 5 was adjusted for unmodifiable risk factors plus rs738409 in PNPLA3. NAFL: non-alcoholic fatty liver; TAT: total adipose tissue, AppAT:

appendicular adipose tissue, GAT: gynoid adipose tissue, AAT: android adipose tissue, VAT: visceral adipose tissue 
Figure legends

Figure 1. ROC curve of WHtR, BMI and the presence of NAFL.

WHtR AUC: 0.823, CI 95\% (0.692-0.955) vs. BMI AUC: 0.720, CI 95\% (0.572-0.955), $\mathrm{p}=0.04$. 


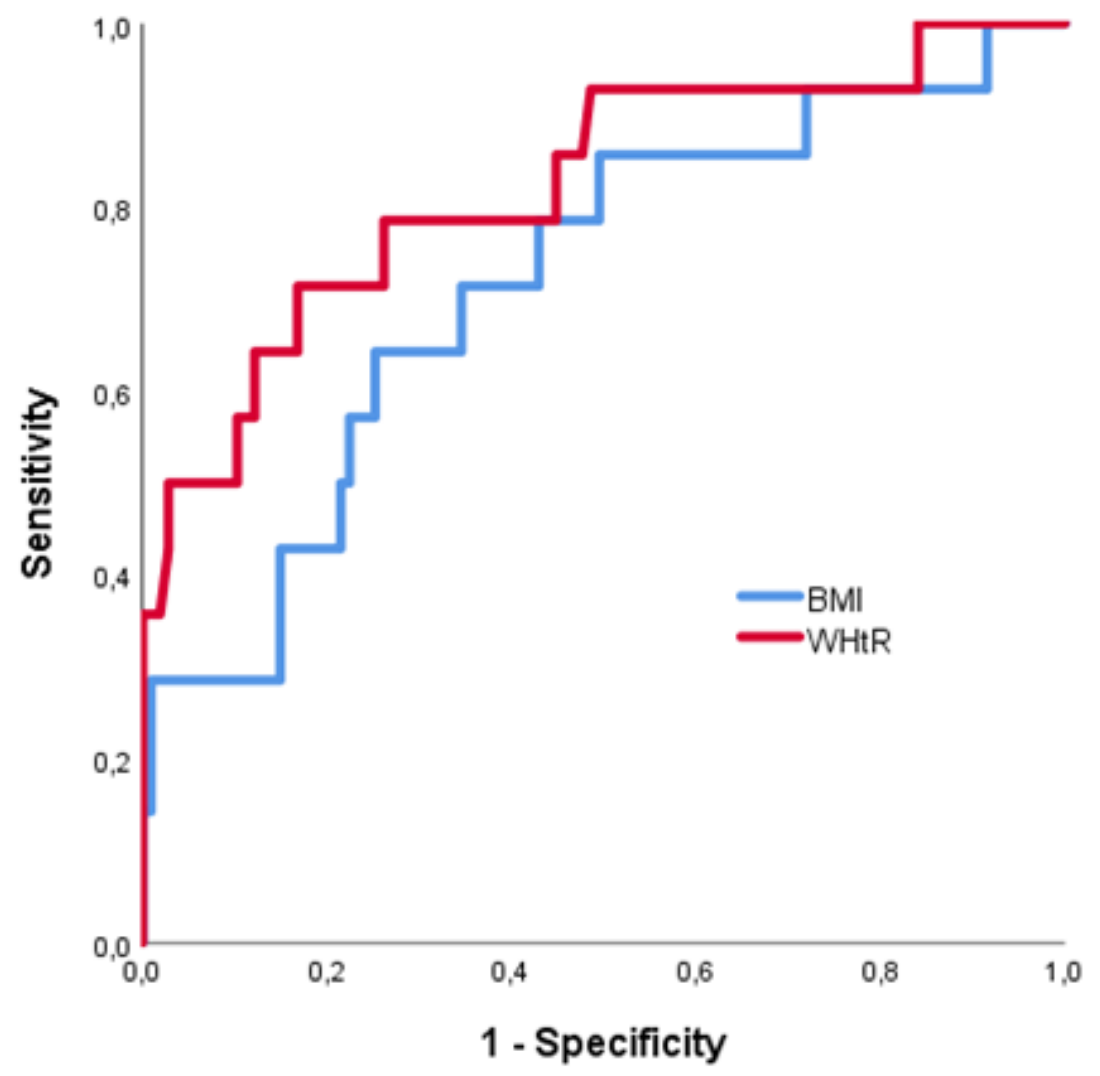

Figure 1. ROC curve of WHtR, BMI and the presence of NAFL.

WHtR AUC: $0.823, \mathrm{Cl} 95 \%(0.692-0.955)$ vs. BMI AUC: $0.720, \mathrm{Cl} 95 \%(0.572-0.955), p=0.04$. 